Mean Places of the. Comparison Stars.

\begin{tabular}{|c|c|c|c|c|c|c|c|c|}
\hline$*$ & BD. & \multicolumn{3}{|c|}{$\alpha 1901.0$} & \multicolumn{3}{|c|}{$\delta 1901.0$} & Authority \\
\hline I & BD. 654 & $4^{h}$ & $8^{r}$ & $5^{5} \cdot 83$ & $+22^{\circ}$ & $27^{\prime}$ & $20 ! 82$ & AG. Berlin 1360 \\
\hline 2 & BD. 657 & 4 & 8 & 37.04 & +22 & 12 & 6.08 & AG. Berlin 1364 \\
\hline 3 & BD. 754 & 4 & 19 & 57.11 & +20 & 54 & $37.9 \mathrm{I}$ & AG. Berlin 1428 \\
\hline 4 & BD. $74^{\circ}$ & 4 & 29 & $51 \cdot 46$ & +19 & 45 & $5^{6.93}$ & A G. Berlin 1228 \\
\hline 5 & BD. 774 & 4 & 39 & $4^{6.97}$ & +19 & 8 & 45.12 & AG. Berlin I 285 \\
\hline 6 & BD. 782 & 4 & $4^{r}$ & 54.88 & +19 & 8 & 29.56 & AG. Berlin 1303 \\
\hline 7 & BD. 734 & 4 & 42 & 53.87 & +18 & $3^{2}$ & $5^{1 \cdot 34}$ & AG. Berlin I 309 \\
\hline 8 & BD. 743 & 4 & 45 & 34.72 & +18 & 40 & 18.39 & AG. Berlin 1326 \\
\hline 9 & BD. 754 & 4 & 49 & $23 \cdot 47$ & +18 & 16 & 55.73 & AG. Berlin 1347 \\
\hline 10 & BD. 688 & 4 & $5^{8}$ & 2 I. II & +16 & 59 & 13.20 & AG. Berlin I $38 \mathrm{I}$ \\
\hline I I & BD. 697 & 5 & I & 18.08 & +16 & 41 & 47.83 & AG. Berlin I 397 \\
\hline 12 & BD. 700 & 5 & 2 & 14.79 & +16 & 50 & 59.04 & AG. Berlin 1401 \\
\hline I 3 & BD. $7 \cdot 5$ & 5 & 5 & $7 \cdot 5 \mathrm{I}$ & +16 & 18 & 45.80 & AG. Berlin 1418 \\
\hline 14 & BD. 795 & 5 & 15 & $5^{8.34}$ & +15 & 45 & 0.78 & AG. Berlin 1481 \\
\hline 15 & BD. 917 & 5 & 22 & 13.20 & +14 & 55 & 53.01 & AG. Leipzig 1531 \\
\hline 16 & BD. 933 & 5 & 25 & 52.07 & +14 & 34 & 9.04 & AG. Leipzig 1671 \\
\hline 17 & BD. 971 & 5 & 39 & 27.22 & +13 & $3^{2}$ & 0.73 & AG. Leipzig 178 г \\
\hline
\end{tabular}

Cordoba Observatory, I 90 I June 22.

Fohn M. Thome.

\title{
Ueber die Beobachtung einer Nova in Bootes im Jahre 1877.
}

(Auszug aus mehreren Schreiben an den Herausgeber).

Bei der in Januar 1877 begonnenen Beobachtungsreihe von 34 Bootis, kam d Bootis in den Verdacht geringer Veränderlichkeit. Um einen passenden Vergleichstern zu haben, zeichnete ich in dem damals gebrauchten Littrow' schen Hinmelsatlas, Blatt 7 , einen gleich hellen Nachbar mit der Bezeichnung $d^{\prime}$ ein. Da mir seiner Zeit der Heis' sche Atlas nicht zur Verfügung stand, war das Fehlen von $d^{\prime}$ in der unvollständigen Littrow'schen Karte nicht auffallend.

Werden nach den im Jahre 1877 erbaltenen Vergleichungen, für die nahen Sterne folgende, der Potsdamer Durchmusterung möglichst angeschlossene Grössen angenommen: $\sigma$ Bootis $=4.85,70$ Virginis Fl. $=5.10, \mathrm{~d}$ Bootis $=5.15$, so ergaben sich für $d^{\prime}$ folgende Helligkeiten (Zeitangaben in M. Z. Marburg a. L.):

\begin{tabular}{|c|c|c|c|c|}
\hline \multicolumn{2}{|c|}{ I 877} & \multicolumn{2}{|r|}{ Gewicht } & Grösse \\
\hline Mai & $3^{\circ}$ & I $1^{h}: 1$ & I & 5.24 \\
\hline Juni & I & 10.9 & $3 / 4$ & .5 .40 \\
\hline & 2 & I I . 5 & I & $5 \cdot 3 I$ \\
\hline & 9 & 12.0 & 1 ? & 5.11 \\
\hline & IO & I 1 . 3 & I & 5.02 \\
\hline & I I & I I. 3 & $8 / 4$ & $5 \cdot 33$ \\
\hline & 12 & $11 \cdot 5$ & $1 ?$ & 5.12 \\
\hline & 13 & I 1.4 & $8 / 4$ & 5.10 \\
\hline & I 5 & I I . 2 & $\mathbf{I}$ & 4.99 \\
\hline & 16 & 11.2 & I & $4.9^{8}$ \\
\hline & 17 & I 2.1 & I & 5.10 \\
\hline Juli & 5 & I I.O & $1 / 2$ & $5 \cdot 3 \mathrm{I}$ \\
\hline & 7 & I I. I & $8 / 4$ & 5.05 \\
\hline & I 4 & I I. 2 & $1 ?$ & 5.01 \\
\hline
\end{tabular}

Die bisherigen Beobachtungen von 34 Bootis u. d. a. geschahen mit blossem Auge; hierbei verursachte der Mond. schein so empfindliche Lücken, dass von. Mitte Juli ab ein kleines galileisches Fernrohr gebraucht wurde. In diesem stand $\mathrm{d}^{\prime}$ wohl etwas zu weit von $34 \mathrm{ab}$, und da sich nähere Vergleichsterne nicht fanden, so fehlen weitere Notizen. Höchst wahrscheinlich war aber der Stern bei vereinzelten, späteren Beobachtungen mit freiem Auge vorhanden, da sein Verschwinden sonst wohl bemerkt worden wäre.

Schluss der Vergleichungen von 34 Bootis 1877 Oct. 10.

Das Verschwinden des Sterns fiel mir zuerst am 9. Jan. I 878 auf. In der Folge wurde die Gegend mit dem I zöll. Fernrohr überwacht und die Grenze (7.-8. Grösse) angegeben, welche der Stern bei einer erneuerten Lichtzunahme nicht hätte überschreiten können, ohne bemerkt zu werden. Im Februar und März 1879 , ferner im Juni 1882 und 1883 wurden mit dem 4 zöll. Fernrohr der Marburger Sternwarte und mit Hülfe der BD. Karten der Umgebung des Sterns angelegt, die mir weiterhin als Grundlage der bis jetzt ohne Ergebniss verlaufenen, freilich recht lückenhaften, Nachforschungen gedient haben. Neuerdings bin ich durch die Freundlichkeit des Herrn Prof. Dr. Küstner in den Besitz zweier am 8. Juli und 8. August dieses Jahres auf der Bonner Sternwarte von Herrn Prof. Mönnichmeyer aufgenommenen Platten der betreffenden Gegend gekommen, die jedenfalls für weitere Untersuchungen von grossem Werthe sein werden.

$\mathrm{Zu}$ Anfang des Jahres 1876 , vielleicht auch schon Ende 1875 zeichnete ich in Riedig's kleiner Ausgabe von Bode's Uranometrie, Karte XIV, in Theilen des Bootes, der Wage und der Schlange alle kleineren Sterne ein. In der Umgebung der Nova sind alle Sterne gezeichnet, während die Nova selbst fehlt, ajso nicht heller als 6.-7. Gr. gewesen sein kann. 
Es sei noch bemerkt, dass der Ort des Sterns innerhalb der Grenzen der Unsicherheit der Zeichnung auf BD. $+21.26069 \mathbf{m}_{4}$ trifft. Dieser Stern wurde im 4 Zöller der Marburger Sternwarte im Februar und März $1879=9.8$, $+21: 2608=9.9$ geschätzt, während auf der Bonner Platte $+21: 2606$ auffallend lichtschwächer als 2608 ist.

Ferner möchte ich noch auf zwei schwache Sterne hinweisen, die ich in meinen Tagebuichern als $f_{2}$ und $f_{3}$ bezeichnet habe, und die veränderlich zu sein scheinen. Beide

Ilmenau, August r 901 .

Zusatz des Herausgebers. Die eingehenden Mittheilungen des Herrn F. Schwab, die hier nur zum Theil als Ergänzung zu Nr. 37 ro wiedergegeben sind, lassen es als ziemlich unzweifelhaft erscheinen, dass 1877 in Bootes thatsächlich ein Stern 5. Gr., der weder vorher noch später in dieser Helligkeit sichtbar war, beobachtet worden ist. $\mathrm{Zu}$ nächst dürfte es wohl angezeigt sein, die Sterne $+21 \% 2606$ und $f_{3}$ weiter zu verfolgen, und sie wo möglich, soweit die Helligkeit es zulässt, auch spectroskopisch zu beobachten. Auf meine Bitte hat Prof. $F$. Deichmiiller die Originale der BD. geprüft und mir unter dem 30 . Juli folgende Mittheilung zukommen lassen: "In den BD.-Zonen finden sich in der Gegend von Schwab's Stern keine vereinzelten Beobachtungen, die auf ein anderes Object als BD. +21.2606 zu deuten wären. Die Beobachtungen des letzteren geben der Vermuthung der Veränderlichkeit einige Unterlage. Der Stern stehen mit BD. $+219_{2} 608$ in gleicher Decl.: $f_{2}$ (10. I I. Gr.) folgt $40^{\mathrm{s}}$ und $f_{3}\left(13^{1} / \mathrm{m}^{\mathrm{m}}\right)$ folgt $43^{\mathrm{s}}$ auf den genannten BD.Stern. Den Stern $f_{3}$ habe ich z. B. 1882 Juni 6 in einem Vierzöller als $9^{\mathrm{m}} \cdot 9$ geschätzt, während er in April und Mai I 883 I $^{\mathrm{m}}$ erschien und jetzt für den damals mir $2 u r$ Verfügung stehenden Vierzöller unsichtbar sein dürfte.

An Interessenten gebe ich auf Wunsch gern eine Blaupause der nach den Bonner photographischen Aufnahmen gezeichneten Karte, nebst Verzeichniss der Grössen ab.

\section{Fr. Schzoab.}

fehlt nämlich in der Schönfeld'schen Zone I 37 I 853 Mai 2, obwohl gerade diese Gegend der Zone sehr sternarm ist und auch der Luftzustand gut war; dagegen ist der Stern (offenbar wegen dieses Fehlens) von Argelander 1858 April 20 im Meridian, an drei Fäden, beobachtet; geschätzt $9.10^{\mathrm{m}}$ bis schwach 9.10m. In den Sucherzonen ist er von Krueger in Z. 435 I 854 April 24 gleich $9.10^{\mathrm{m}}$ beobachtet, obwohl die Luft sehr dunstig war. Der Stern könnte aber an diesem Abend auch etwas heller gewesen sein, denn Krueger bemerkt, dass die Stellung des Oculars bei dieser Zone geändert worden war, so dass bei dem entschieden veränderten Aussehen der Sterne gegen früher ein beträchtlicher Unterschied in der Schätzung der Grössen sich zeigen mag. Zu schwache Schätzungen von $1 / 2$ Grössenclasse gegenüber den anderen Zonen kommen auch mehrfach in Zone 435 vor«.

$$
K r \text {. }
$$

\section{Neue veränderliche Sterne.}

In ^Report of His Majesty's Astronomer at the Cape of Good Hope for the year I goo werden vier veränderliche Sterne mitgetheilt, die $R . T$. A. Innes im verflossenen Jahre bei Gelegenheit der Revision der C. Ph. DM. entdeckt hat. Einer von diesen findet sich schon A. N. 3679 als Var. 25.1900 Octantis aufgeführt; die drei übrigen sind die folgenden:

Var. 27.1900 Volantis (Cord. 2C. $\left.7^{\mathrm{h}} 2258\right) \quad 7^{\mathrm{h}} 3 \mathrm{I}^{\mathrm{m}} \cdot 9-73^{\circ} 7^{\prime}$ (1875) Gr. 9.1 - unsichtbar Periode $400^{\mathrm{d}} \pm$

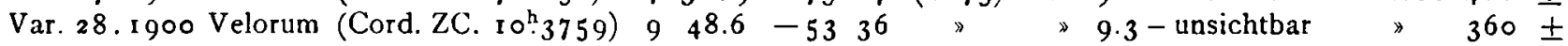

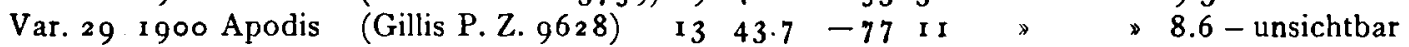

Der erste Stern, Cord. ZC. $7^{\text {h }} 225^{8}$, gehört zu den Zonensternen, welche in Cordoba bei der Revision nicht aufgefunden sind (vgl. Cord. ZC. I. Band p. XV).

$K r$.

Notiz. Am 22. September ist auf der Insel Hven unter Anwesenheit des Königs von Schweden und der Vertreter der dänischen und schwedischen Universitäten eine Gedenkfeier zu Ehren Tycho Brahe's abgehalten worden.

$K r$.

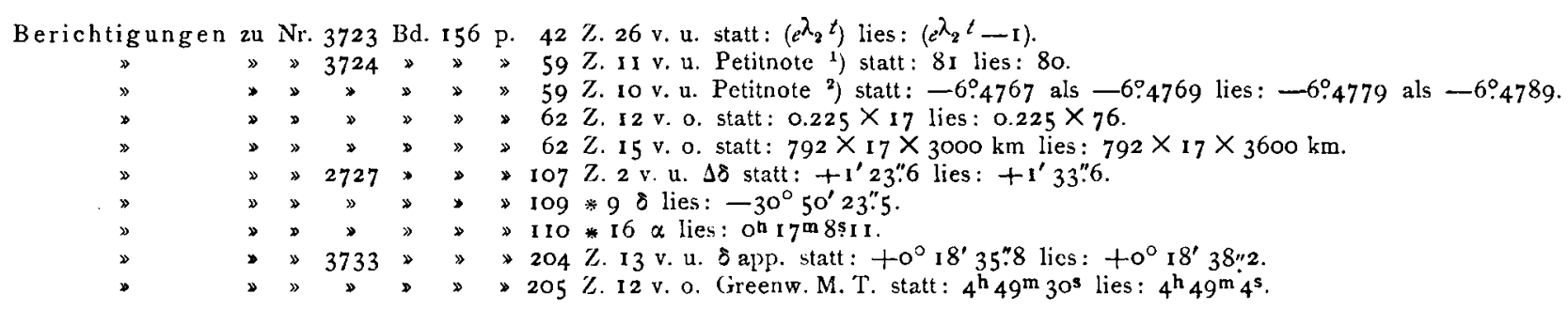

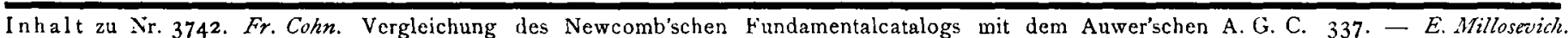
Osservazioni di (433) Eros. 345. - 7. M. Thome. Cordoba Observations of (433) Eros. 347. - Fr. Schwab. Ueber die Beobachtung einer Nova in Bootes im Jahre 1877. 349. Z Zusatz hicrzu. 35 I. - Neue veränderliche Sterne. 35 t. Notiz. 35I. - Berichtigungen. 351 . 